\title{
Le mie ricerche sulla Laurea di Paracelso ${ }^{1}$
}

\author{
Nota riassuntiva sul loro stato attuale \\ Per il Dott. Isidoro Ghibellini, Rovigo
}

Come è noto ai componenti di questa studiosa Assemblea gli antiparacelcisti hanno affermato: PARAgelso è il più grande impostore del millecinquecento, il ciarlatano che è riuscito a farsi credere medico e medico di non comune valore; $i$ paracelsisti hanno risposto: Paracelso è un maestro divino, un medico che ha superato tutte le scuole e tutti gli insegnamenti, che ha lasciato la sua impronta rivoluzionaria in ogni campo della scienza (Мгото).

Dopo due anni di ricerche eseguite presso la Biblioteca Ariostea, presso gli archivi notarile, vescovile e universitario di Ferrara, presso l'Accademia dei Concordi di Rovigo, presso la biblioteca universitaria di Bologna, presso l'Archivio di Stato di Modena, Salerno, Padova e Venezia, nonchè presso l'Archivio Municipale di Lonigo, dopo aver letto molte pubblicazioni sulla vita e sulle opere di Paragelso, vorrei sintetizzare la mia fatica con le seguenti affermazioni:

1. Il padre di Paracelso era medico e un medico di indubbia competenza, dedito anche alle studio della botanica, allo studio ed all'esercizio pratico della chimica e fu il primo ed il più prezioso maestro del figlio, che da lui apprese molte nozioni e l'avviamento nelle predette tre branche della medicina, botanica e chimica.

2. Secondo alcuni studiosi Paragelso assunse questo nome dopo essersi laureato a Ferrara e secondo altri, e con maggior probabilità, quando egli nel 1510 entrò diciasettenne nel collegio o scuola superiore di Basilea, ove era costume latinizzare i cognomi.

3. Ł̀ dato per certo dagli storici che egli fu allievo del benedettino Grovann Heidemberg, latinizzato in Tritemio, versatissimo nelle scienze occulte, e di Scheit vescovo di Stettgach e di Matteo Schacht suffraganeo di Freisingen; che nel 1515 lavorava o indagava nelle miniere dei FugGer a Schwatz; che si recò a Vienna, ove esiste tutt'ora una sua ricetta, a Colonia, a Parigi e a Montpellier; che dalla Francia passò in Italia, prima a Bologna indi a Padova e poi a Ferrara, ove pare si addottorasse; che visitò Salerno, il Portogallo, la Spagna, in Inghilterra l'università di Oxford; che nel 1522 era a Venezia quale chirurgo militare dell'armata nella guerra tra Francesco I ${ }^{0}$ e CARLO Vo ${ }^{0}$

4. L'esito felice della cura praticata al noto editore FröBEN, ingigantì la sua grande fama di terapeuta.

5. Nel 1526 resisi vacanti i posti di medico comunale o di chirurgo a Basilea e di

1 Communicazione al Convegno storico-medico, Bologna, 26-27 aprile 1952. 
professore in quella università è chiamato dal Magistrato a coprirli. Gli altri professori universitari si opposero a che PARAcelso insegnasse perchè non aveva superato gli esami difficili da essi sostenuti ma il Magistrato dopo aver esaminato la posizione confermò Paracelso anche nell'insegnamento. Le sue lezioni furono clamorose ed ebbero enorme successo perchè erano dette in tedesco, perchè negavano o criticavano tutta la medicina antica, perchè alla medicina dava un indirizzo rivoluzionario, perchè traeva vita dalla observatio et ratio e perchè PARAcelso osò bruciare davanti alle porte dell'università $i$ testi di Galeno e di Avicenna.

6. Nel processo col canonico di S. Chiara, Cornelio di Lichtenfels, Paracelso giurò di essersi laureato nell'alta scuola di Ferrara, ovvero giurò sulla laurea conseguita a Ferrara.

Un fatto così importante per il nostro argomento non è però ben chiaro nei dati storici pervenutici. Infatti è a chiedersi, perchè gli fu richiesto di giurare dal tribunale? Perchè dicesse la verità nella controversia col canonico o perchè non fu in grado di presentare l'attestato di laurea chiestogli in quanto al tribunale era stato avanzato il dubbio che egli non fosse medico ? Ovvero gli fu domandato di dire la verità giurando sull'attestato di laurea?

La cosa ha naturalmente molta importanza per l'indagine che stiamo conducendo e purtroppo nonostante le richieste fatte sia alla biblioteca di Salisburgo che all'università di Basilea non sono riuscito ad avere elementi che possano comunque portare luce su tale giuramento. Qualche autore asserisce anche che egli ricordò solo davanti ai giudici il giuramento fatto a Ferrara in occasione del suo dottorato.

Comunque stia il fatto è indubbio che direttamente o indirettamente con quel giuramento egli affermò davanti a Dio e agli uomini di essersi laureato nell'università di Ferrara.

E questo giuramento credo sia stato finora l'unico argomento che ha fatto asserire a tanti studiosi che egli si sia effettivamente laureato a Ferrara. Come vedremo è un argomento che non ne da però la prova assoluta.

7. Nel manifesto che il Paracelso pubblicò in occasione della sua nomina a medico della città ed a professore della università di Basilea egli si definisce doctor utriusque mediacine.

8. Secondo il Mrotto Paracelso ottenne cariche importanti in base non al diploma universitario ma bensì in seguito alla raccomandazione del FröBEN.

9. Nella lettera che egli scrive ad Erasmo da RHotterdam offre all'amico se stesso medico e le medicine. - Nella risposta Erasmo lo definisce medico di grande celebrità e portentoso nelle cure.

10. Nel 1526 è a Strasburgo come medico ma egli non si inscrive però alla corporazione dei medici ma bensì in quella dei mercanti di grano e dei mugnai (Miотто). Perchè? 
11. In tutte le sue opere, stampate in gran parte dopo la sua morte, appare sul frontispizio o il suo titolo di medico o quello di maestro o di professore.

12. Nell'epitafio della sua tomba il titolo di medico e di terapeuta eccezionale appare in modo evidente.

13. Tutti gli studiosi, meno gli antiparacelsisti giurati, lo chiamano medico e maestro insigne e di tale valore da rivoluzionare la medicina del 1500.

14. Le sue opere ci attestano indiscutibilmente che egli aveva una cultura medica molto vasta.

15. È dato per certo che egli ha occupato il posto di chirurgo militare nei Paesi Bassi e al soldo dei Veneziani (1522). A questo proposito è da ricordare che chi faceva il chirurgo in via generale non era medico.

16. Paracelso aveva promesso ad Oporino, suo segretario, di insegnargli la medicina nello spazio di due anni. Ora è qui da considerare che se Oporino, il tedesco Giovanni Herbst, persona di cultura in quanto dapprima correttore di bozze alla stamperia FröBen, poi rettore del ginnasio di Basilea e professore di greco all'Accademia di quella stessa città, si decise di seguire PARACELSo per imparare da lui la medicina bisogna ritenere che egli lo considerasse medico.

17. Questo viene a confermare un fatto di per se evidente e cioè quanto, Giondano Bruno, vissuto subito dopo, disse di Paragelso: «Paracelso disponeva di una conoscenza delle scienze mediche e dei rimedi più profonda di GaLENo, di Avicenna e di tutti i medici», anche se il cancelliere Francesco Bacone da Verulamio il suo primo accusatore (22 gennaio 15618 aprile 1626) lo abbia chiamato figlio adottivo degli asini, mentre invece il REBER nella sua monografia, disse di lui che nel secolo XVI non esistè uomo più saggio, mentre gli storici imparziali, lo considerarono un uomo assennato e coraggioso che colla sua chiaroveggenza e cultura rese grandi servizi all'umanità per avere grandemente contribuito alla diffusione della scienza medica svincolandola dalle pastoie e dai dettami dei medici antichi, introducendo in terapia molti preparati importanti, dando un indirizzo completamente nuovo rivoluzionario anche alla chimica ed in entrambe sostenendo la base sperimentale.

18. Nella chirurgia egli eccelse; cominciò a curare le ferite col sale e meritò anche la citazione di Cesare Magati.

19. Le mie ricerche fatte a Ferrara e altrove finora hanno avuto esito negativo in quanto non ho trovato neppure annotato uno dei vari nomi di Paracelso. Le varie storie di Ferrara non vi fanno anche il più fugace accenno tranne quella recente del Visconti, il quale però, come tutti gli altri studiosi che l'asseriscono, non ne cita la fonte storica comprovante; di PARAgelso non si trova alcuna postilla o nota nè fra le matricole nè fra gli attestati notarili delle lauree concesse in quel periodo. 
20. A proposito però delle matricole o inscrizioni degli studenti e delle lauree allora concesse vi sono molte osservazioni da avanzare perchè le matricole non erano sempre regolarmente registrate.

21. Le lauree erano conferite o con una certa pompa e dietro un esame pubblico davanti i componenti il Collegio dei medici fisici ove il candidato poteva essere assistito da uno dei suoi professori ovvero erano conferite privatamente dai singoli professori; in quest'ultimo caso si chiamavano licenze e naturalmente difficilmente ne era convalidato l'atto dal notaio e raramente ne era tenuta registrazione; questo stato di cose lascia intravedere quali deviazioni o abusi ne potessero nascere e come era materialmente impossibile controllare le inscrizioni all'università e le lauree concesse.

Queste deduzioni sono state tratte da lettere, documenti manoscritti di epoca posteriore al 1500 ma è probabile che tali abusi perdurassero da tempo, poichè ovunque, e quindi non solo a Ferrara, la medicina era esercitata da molti praticoni senza il dottorato, la chirurgia anche nei secoli successivi era fatta da infermieri, da norcini, da barbieri, i quali al massimo avevano assistito qualche medico nell'esercizio della sua professione. Però il secolo che va all'incirca dal 1450 al 1550 fu quello del maggior splendore dello studio Estense tanto che esso era considerato se non la prima almeno una delle prime università di quel tempo: probabilmente per questo Paracelso la frequentò e per questo forse ambì laurearvisi. Il fatto si intonerebbe evidentemente al suo abito mentale e la sua ripetuta asserzione «Alterius non sit, qui suus esse potest» lo confermerebbe.

22. Un'altra osservazione debbo fare e stavolta sull'incarico di notaio segretario presso detta università. È stato detto che tale incarico ogni Facoltà lo affidava ad un proprio notaio. Ma il VentuRINI noto e valoroso conservatore dell'archivio notarile di Ferrara, asserisce che tante e tante sono le matricole e le attestazioni di laurea rilasciate nel 1500 che è ben difficile comprendere come potessero essere rilasciate da un solo notaio. Quindi probabilmente per quanto ufficialmente ogni facoltà avesse il suo notaio segretario, ciò non di meno non doveva essere inibito fare vistare le suddette attestazioni da altri notai e dal controllo fatto sull'apposito registro ho potuto constatare che $i$ notai che rogarono in Ferrara nel decennio 1513-1522 furono ventitre.

23. Infine ancora una osservazione sul lavoro del PARDI, Titoli dottorali conseguiti presso l'università di Ferrara nel 1400-1500. Mi sono provato di controllare le matricole di inscrizione e lauree inerenti al periodo del mio studio. $\mathbf{E}$ una fatica improba che oltre il resto non può dare dei risultati attendibili,

$1^{\circ}$ perchè occorrerebbero anni di pazientissimo lavoro per leggere le attestazioni sopradette;

$2^{\circ}$ gli atti dei vari notai sono tutt'altro che completi; per esempio fra gli atti del Notaio Matteo CaPrili che pare avesse l'incarico di segretario 
della facoltà medica mancano ben 49 pagine concernenti proprio il decennio da me preso in esame;

$3^{\circ}$ inoltre coma abbiamo accennato non sempre gli esami di laurea erano pubblici ma molte volte gli stessi professori universitari singolarmente rilasciavano licenze che probabilmente non erano poi neppure registrate dal notaio.

Ciò premesso mi permetto di dubitare che il suddetto elenco del PARDI possa essere completo.

Volendo ora fare il punto sulle mie ricerche dovrei dire:

a) che non sono stati reperiti documenti comprovanti o neganti la laurea di Paracelso a Ferrara o altrove; l'unico documento del suo soggiorno in Italia potrebbe essere, se del caso, un suo ritratto esistente a Venezia;

b) che il maggior contributo positivo per tale laurea permane a tutt'oggi il giuramento di Paracelso al tribunale di Basilea: il valore del giuramento è innegabile ma non può costituire una base di assolutezza;

c) che Paracelso fosse addottorato sembrerebbe comprovarlo anche il fatto che il Magistrato di Basilea nonostante il ricorso avanzato dagli altri professori dell'università, lo confermò al posto di medico della città qualora però fosse accertato che egli ebbe questo incarico e non quello di chirurgo come alcuni invece asseriscono;

d) che però l'incarico di professore universitario non comportava il titolo dottorale nè allora nè adesso tanto è vero che pure a Ferrara era conferito anche a studenti;

e) che le ricerche archivistiche eseguite dal Pardi, dallo Strunz e da altri che possono comprovare o negare la laurea di PARAcelso perchè incomplete;

f) che Paracelso era fornito di vastissima cultura medico chirurgica e terapeutica originata dalla sua avidità senza pari di bere a tutte le fonti del sapere (Мготт);

g) che egli quando giunse a Ferrara aveva già visitato e frequentato parecchie università ed aveva già una vasta cultura medica, certamente uguale ma facilmente superiore a quella degli altri laureandi e che perciò, come CoperNICO, poteva essere ritenuto maturo pel dottorato;

h) che egli non del tutto immeritatamente fu chiamato dottore, maestro e professore in quanto fu una delle persone più colte del suo tempo;

i) che pertanto egli meritò questi titoli e se pur egli non ebbe a sottostare, come è probabile, alla formalità dell'esame e del conferimento della laurea ciò non di meno per $i$ suoi indiscussi requisiti, qualora potesse risorgere come aveva promesso e presentarsi a questa preclara assemblea di studiosi, gli si potrebbe pure a lui conferire una laurea ad onorem, in quanto se è vero che non ne possiamo documentare il dottorato, è altrettanto vero che non possiamo neppure dimostrare che egli abbia giurato il falso. 